\title{
Dynamics of Intracellular Polymers in Enhanced Biological Phosphorus Removal Processes under Different Organic Carbon Concentrations
}

\author{
Lizhen Xing, ${ }^{1}$ Li Ren, ${ }^{1}$ Bo Tang, ${ }^{2}$ Guangxue Wu, ${ }^{2}$ and Yuntao Guan ${ }^{2}$ \\ ${ }^{1}$ School of Municipal and Environmental Engineering, Shandong Jianzhu University, Jinan, Shandong, 250101, China \\ ${ }^{2}$ Key Laboratory of Microorganism Application and Risk Control (MARC) of Shenzhen, Graduate School at Shenzhen, \\ Tsinghua University, Shenzhen, Guangdong, 518055, China
}

Correspondence should be addressed to Guangxue Wu; wu.guangxue@sz.tsinghua.edu.cn

Received 7 October 2013; Accepted 16 November 2013

Academic Editor: Kannan Pakshirajan

Copyright (C) 2013 Lizhen Xing et al. This is an open access article distributed under the Creative Commons Attribution License, which permits unrestricted use, distribution, and reproduction in any medium, provided the original work is properly cited.

\begin{abstract}
Enhanced biological phosphorus removal (EBPR) may deteriorate or fail during low organic carbon loading periods. Polyphosphate accumulating organisms (PAOs) in EBPR were acclimated under both high and low organic carbon conditions, and then dynamics of polymers in typical cycles, anaerobic conditions with excess organic carbons, and endogenous respiration conditions were examined. After long-term acclimation, it was found that organic loading rates did not affect the yield of PAOs and the applied low organic carbon concentrations were advantageous for the enrichment of PAOs. A low influent organic carbon concentration induced a high production of extracellular carbohydrate. During both anaerobic and aerobic endogenous respirations, when glycogen decreased to around $80 \pm 10 \mathrm{mg} \mathrm{C}$ per gram of volatile suspended solids, PAOs began to utilize polyphosphate significantly. Regressed by the first-order reaction model, glycogen possessed the highest degradation rate and then was followed by polyphosphate, while biomass decay had the lowest degradation rate.
\end{abstract}

\section{Introduction}

Eutrophication means the overgrowth of algae and cyanobacteria, and, after their death, it causes water pollution by the depletion of oxygen and the release of toxins. Phosphorus in discharged wastewater is one of the main elements contributing to eutrophication and the worsening water quality. In order to reduce or control eutrophication of water bodies, enhanced biological phosphorus removal (EBPR) has been applied widely for phosphorus removal from wastewaters [1]. In EBPR, alternative anaerobic and aerobic phases are adopted and polyphosphate accumulating organisms (PAOs) with excess phosphorus accumulation ability will be enriched [1]. During the anaerobic phase, PAOs take up organic carbons such as acetate and propionate and store them as intracellular polymers such as poly- $\beta$-hydroxybutyrate (PHB), with polyphosphate as the energy source and glycogen as the reducing power source [1]. In the liquid, the concentration of organic carbon decreases while the concentration of phosphate increases. During the aerobic phase, PAOs synthesize new organisms, restore polyphosphate, and replenish glycogen with stored $\mathrm{PHB}$ as the energy and organic carbon sources [1]. In the liquid, phosphorus concentration decreases. PAOs can accumulate polyphosphate with proportions to the total dry biomass weight in the range of $4-15 \%$, which is much higher than that of $2 \%$ of general microorganisms [2]. Phosphorus will be removed from wastewater by removing residue activated sludge with high phosphorus content from the wastewater treatment system.

Polyphosphate, PHB, and glycogen are three important polymers of PAOs in EBPR, and the accurate analysis of these components plays an important role in elucidating their dynamics in EBPR. For polyphosphate and PHB, there are some well-recognized analytical methods, while for glycogen, usually, the carbohydrate in activated sludge is converted to glucose under acidic conditions by heating and then analyzed by high-pressure liquid chromatography (HPLC) or spectrophotometer [3]. By this method, the concentration of 
glycogen inside the biomass will be overestimated by around $40 \%$ [3], because carbohydrate in the biomass includes not only intracellular glycogen but also extracellular carbohydrate in extracellular polymeric substances (EPS). Therefore, so as to accurately describe dynamics of intracellular polymers of PAOs, intracellular and extracellular polymers should be differentiated.

During weekend and wet seasons, EBPR may experience deterioration or even failure due to the overflow or the low organic carbon concentration, and this phenomenon is named as the "monday peak" $[4,5]$. Ahn et al. [6] found that, after being shocked from the low organic carbon, 20 days were required for PAOs to recover to the normal condition. How to ensure the stable operation of EBPR during low organic carbon conditions is one of the important tasks for wastewater treatment. Performance of EBPR depends on not only the amount of PAOs acclimated but also on the polymers of PAOs due to their important function during biochemical metabolism of PAOs. Most of previous studies have focused on the dynamics of PHB [7] while less focused on glycogen or polyphosphate. As mentioned above, glycogen and polyphosphate also play very important roles in EBPR. Therefore, for further studies, it is necessary to examine dynamics of all these polymers of PAOs in EBPR studies. Under adequate organic carbon conditions, microorganisms will experience excess biomass production and endogenous respiration is not obvious. However, under endogenous conditions, biomass production will be affected and endogenous respiration of PAOs may be dominated. For polymer dynamics under endogenous respiration conditions, there are some contrary conclusions. Lopez et al. [8] obtained that PAOs degraded polyphosphate during the initial several days, while Yilmaz et al. [9] obtained that polyphosphate was released within one day. Therefore, examining dynamics of polymers of PAOs is an important aspect to maintain activities of PAOs $[10,11]$, and this should be further investigated.

In this study, metabolism of PAOs and dynamics of polymers under different organic carbon concentrations were examined so as to elucidate the function of polymers in EBPR. In addition, dynamics of polymers under endogenous respiration conditions was also investigated to provide some clues for controlling and adjusting the EBPR during low organic carbon shocking conditions.

\section{Materials and Methods}

2.1. PAOs Acclimation. PAOs were acclimated in two sequencing batch reactors (SBRs) with different influent organic carbon concentrations at $25^{\circ} \mathrm{C}$. One SBR (SBR-L) was supplied with a low sodium acetate $(\mathrm{NaAc})$ concentration (chemical oxygen concentration, named as COD, of around $200 \mathrm{mg} / \mathrm{L}$ ) and the other SBR (SBR-H) with a high NaAc concentration (COD of around $400 \mathrm{mg} / \mathrm{L}$ ). The effective SBR working volume was $6 \mathrm{~L}$ and the SBR phases of fill, mixing, aeration, settlement, and withdrawal were controlled by timers. The SBRs were operated 4 cycles per day and each cycle included $120 \mathrm{~min}$ of anaerobic phase with $10 \mathrm{~min}$ of fill phase, $180 \mathrm{~min}$ of aerobic phase, 40 min of settlement phase, and idle/withdrawal of $20 \mathrm{~min}$. During the fill phase, influent wastewater

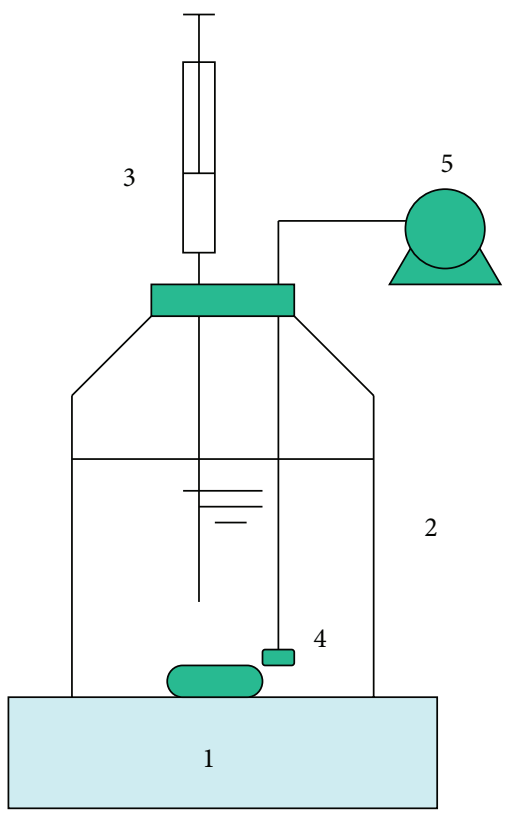

FIgURE 1: Diagram of the batch experiment reactor. (1) Magnetic stirrer; (2) reactor; (3) liquid sampler; (4) aeration stone; (5) air pump. 4 and 5 only worked during the aerobic condition.

of $3 \mathrm{~L}$ was pumped by peristaltic pumps into the reactor. Each day, at the same time before the settlement phase, $600 \mathrm{~mL}$ of mixed liquor was withdrawn from the reactor to maintain the sludge retention time of around 10 days. The SBRs were seeded with activated sludge taken from Nanshan wastewater treatment plants in Shenzhen, China.

Synthetic wastewater was treated in both reactors. The synthetic wastewater for SBR-L was comprised of NaAc of $255 \mathrm{mg} / \mathrm{L}, \mathrm{Na}_{2} \mathrm{HPO}_{4}$ of $91.6 \mathrm{mg} / \mathrm{L}, \mathrm{NH}_{4} \mathrm{Cl}$ of $76.5 \mathrm{mg} / \mathrm{L}$, $\mathrm{CaCl}_{2} \cdot 2 \mathrm{H}_{2} \mathrm{O}$ of $14 \mathrm{mg} / \mathrm{L}, \mathrm{MgSO}_{4} \cdot 5 \mathrm{H}_{2} \mathrm{O}$ of $90 \mathrm{mg} / \mathrm{L}$, trace elements of $0.12 \mathrm{~mL} / \mathrm{L}$, and yeast extract of $10 \mathrm{mg} / \mathrm{L}$. For the SBR-H, only the NaAc concentration was changed to $510 \mathrm{mg} / \mathrm{L}$, while other components were the same as those of the SBR-L. The components of trace elements were made according to those of Smolders et al. [12].

2.2. Batch Experiments. Batch experiments were carried out at $25^{\circ} \mathrm{C}$ with activated sludge taken from the parent SBRs at steady state. Each batch experiment included activated sludge taken from SBR-H and SBR-L, respectively. For each batch experiment, $400 \mathrm{~mL}$ of mixed liquor was withdrawn from the parent SBRs and then filled in a $600 \mathrm{~mL}$ capped glass flask (Figure 1). On the cap, several ports were made for sampling, aeration, and so forth. The ports were sealed with tubes for taking samples and so forth. Each batch experiment was replicated and only average results were presented.

In order to examine dynamics of polymers of PAOs under anaerobic conditions, excess organic carbon was supplied and dynamics of different parameters were examined including glycogen, $\mathrm{PHB}$, acetate, and orthophosphate $\left(\mathrm{PO}_{4}{ }^{3-}-\mathrm{P}\right)$. At the beginning of the experiment, acetate was supplied to the two batch reactors with the initial concentration of $1000 \mathrm{mg} / \mathrm{L}$ 
and then capped to start the experiment. Samples were taken at intervals of $10 \mathrm{~min}$ or $15 \mathrm{~min}$.

Endogenous experiments were carried out under both anaerobic and aerobic conditions to examine dynamics of polymers of PAOs. For the anaerobic endogenous experiment, activated sludge mixed liquor taken from the parent reactor was placed in glass flasks directly with an initial nitrogen gas purging for 5 minutes. For the aerobic endogenous experiments, activated sludge mixed liquor taken from the parent SBRs was aerated constantly. Samples were taken at intervals of 12 or 24 hours and each endogenous experiment lasted for 168 hours.

2.3. Analytical Methods. Volatile suspended solids (VSS), suspended solids (SS), ammonium nitrogen $\left(\mathrm{NH}_{4}{ }^{+}-\mathrm{N}\right)$, and $\mathrm{PO}_{4}{ }^{3-}$ - $\mathrm{P}$ were measured according to standard methods for the examination of water and wastewater [13].

PHB was measured with the methods of Karr et al. [14] and Rodgers and $\mathrm{Wu}$ [15]. Total carbohydrate of biomass was measured according to Lanham et al. [16]: (a) $2 \mathrm{~mL}$ of mixed liquor sample was added into a glass tube with $1 \mathrm{~mL}$ of deionized water and $0.3 \mathrm{~mL}$ of $6 \mathrm{M} \mathrm{HCl}$ and then mixed; (b) the mixer was digested at $100^{\circ} \mathrm{C}$ for $2 \mathrm{~h}$; (c) after cooling down to room temperature, the digested liquor was centrifuged at $12000 \mathrm{rpm}$ for $2 \mathrm{~min}$, and the supernatant was taken for the measurement of glucose by HPLC. Extracellular carbohydrate was extracted according to the method of $\mathrm{Li}$ and Yang [17] and Sponza [18]: (a) $2 \mathrm{~mL}$ of mixed liquor was heated at $60^{\circ} \mathrm{C}$ for $30 \mathrm{~min}$; (b) the heated samples were then centrifuged at $12000 \mathrm{rpm}$ for $2 \mathrm{~min}$, and EPS was released from the biomass to the supernatant. The extracellular carbohydrate inside the supernatant was then pretreated as that of the total carbohydrate and then analyzed by HPLC.

$\mathrm{PHB}, \mathrm{NaAc}$, and glucose were measured with the HPLC equipment (Shimadzu LC-20A, Japan). PHB and acetate were measured with the UV detector at $210 \mathrm{~nm}$, while glucose was measured with the RID 10-A detector. All these parameters were measured using the Aminex column (HPX-87H, BioRad, USA). The testing conditions used during the HPLC testing were (a) the mobile phase of $0.1 \%$ sulfuric acid at the flow rate of $0.6 \mathrm{~mL} / \mathrm{min}$; (b) the column temperature of $40^{\circ} \mathrm{C}$; (c) the detector cell temperature of $40^{\circ} \mathrm{C}$; (d) the injection volume of $20 \mu \mathrm{L}$ for $\mathrm{PHB}$ and the testing duration of $35 \mathrm{~min}$; while those for glucose of $50 \mu \mathrm{L}$ and $15 \mathrm{~min}$ and for acetate of $20 \mu \mathrm{L}$ and $20 \mathrm{~min}$, respectively.

\section{Results and Discussion}

3.1. Acclimation of PAOs and Dynamics of Polymers in Typical Cycles. By using two different influent acetate concentrations, one with a high influent acetate concentration of SBR$\mathrm{H}$ and the other of SBR-L, after 60 days of acclimation, the two SBRs reached steady state. Under steady state, for SBR$\mathrm{H}$, the SS was $2345 \pm 60 \mathrm{mg} / \mathrm{L}$, VSS was $1725 \pm 68 \mathrm{mg} / \mathrm{L}$, and the effluent $\mathrm{PO}_{4}{ }^{3-}$-P was $5.42 \pm 0.4 \mathrm{mg} / \mathrm{L}$, while for SBR-L, the SS was $1320 \pm 50 \mathrm{mg} / \mathrm{L}$, VSS was $930 \pm 56 \mathrm{mg} / \mathrm{L}$, and the effluent $\mathrm{PO}_{4}{ }^{3-}$-P was $8.28 \pm 0.5 \mathrm{mg} / \mathrm{L}$. In spite of the relatively high effluent $\mathrm{PO}_{4}{ }^{3-}-\mathrm{P}$ concentrations in both reactors, which could be due to the high influent $\mathrm{PO}_{4}{ }^{3-}-\mathrm{P}$ concentration applied, a high phosphorus content in the biomass was obtained for both reactors. The phosphorus content inside the dry biomass was $9.2 \%$ in SBR-H and $13.2 \%$ in SBRL. The sludge yield coefficient was $23.6 \mathrm{mgSS} / \mathrm{g}$ COD or $17.5 \mathrm{mg}$ VSS/g COD in SBR-H and was $26.5 \mathrm{mg} \mathrm{SS/g} \mathrm{COD} \mathrm{or}$ $18.6 \mathrm{mg}$ VSS/g COD in SBR-L.

Dynamics of parameters in typical cycles in both reactors are shown in Figure 2. Typical EBPR characteristics were observed in both reactors. In SBR-H, during the anaerobic phase, $\mathrm{PHB}$ reached the peak in the initial $30 \mathrm{~min}$ with the value of $54.1 \mathrm{mg} \mathrm{PHB}-\mathrm{C} / \mathrm{g}$ VSS; the released $\mathrm{PO}_{4}{ }^{3-}-\mathrm{P}$ was $59.6 \mathrm{mg} \mathrm{P} / \mathrm{g} \mathrm{VSS}$ and the concentration of glycogen decreased to $53.5 \mathrm{mg} \mathrm{C/g} \mathrm{VSS}$. In SBR-L, in the anaerobic phase, $\mathrm{PHB}$ reached the peak value of $34.1 \mathrm{mg} \mathrm{PHB}-\mathrm{C} / \mathrm{g}$ VSS at minute 45 ; the released $\mathrm{PO}_{4}{ }^{3-}-\mathrm{P}$ was $64.26 \mathrm{mg} \mathrm{P} / \mathrm{g}$ VSS and the concentration of glycogen decreased to $49.8 \mathrm{mg} \mathrm{C} / \mathrm{g}$ VSS.

In spite of different influent acetate concentrations applied in both reactors, similar sludge yield coefficients were obtained in SBR-H and SBR-L, indicating that sludge production was not significantly affected by the influent organic carbon concentrations. However, a slightly higher phosphorus content was obtained of $13.2 \%$ in SBR-L than that of $9.2 \%$ in SBR-H. In addition, anaerobic phosphorus release potential was similar in both reactors, with values of $54.6 \mathrm{mg}$ P/g VSS in SBR-H and of $58.9 \mathrm{mg}$ P/g VSS in SBR-L. These results showed that, in the anaerobic and aerobic alternating system, a low influent organic carbon concentration favoured the acclimation of PAOs, which could be due to the high competition ability of PAOs compared with their competitors of glycogen accumulating organisms under low organic carbon conditions. Similar results were also obtained by $\mathrm{Tu}$ and Schuler [19].

3.2. Anaerobic Dynamics of Polymers of PAOs with Excess Supply of Organic Carbons. Anaerobic dynamics of polymers of PAOs with excess supply of organic carbons is shown in Figure 3. From Figure 3, it is shown that, from minute 50, a high variation in the concentration of various parameters occurred and the regressed biokinetics of polymers is given in Table 1 with durations from minutes 0 to 50 (Phase A) and from minutes 50 to 115 (Phase B).

After supply with excess acetate, PAOs degraded glycogen for supplying reducing power and partial energy. In SBR-H, the total carbohydrate decreased from $189.8 \mathrm{mg} \mathrm{C/}$ $\mathrm{g}$ VSS to $122.8 \mathrm{mg} \mathrm{C/g} \mathrm{VSS}$ and the intracellular carbohydrate decreased from $108.3 \mathrm{mg} \mathrm{C/g} \mathrm{VSS}$ at minute 0 to $38.9 \mathrm{mg} \mathrm{C/}$ g VSS at minute 50, while the extracellular carbohydrate kept relatively stable at $82.8 \pm 2.2 \mathrm{mg} \mathrm{C} / \mathrm{g} \mathrm{VSS}$; the decreased intracellular carbohydrate concentration was $69.5 \mathrm{mg} \mathrm{C} / \mathrm{g}$ VSS during the whole reaction phase. In SBR-L, the total carbohydrate decreased from $238.0 \mathrm{mg} \mathrm{C} / \mathrm{g}$ VSS to $198.5 \mathrm{mgC} / \mathrm{g} \mathrm{VSS}$ and the intracellular carbohydrate decreased from $145.5 \mathrm{mgC} / \mathrm{g} \mathrm{VSS}$ to $56.0 \mathrm{mg} \mathrm{C} / \mathrm{g} \mathrm{VSS}$, while the extracellular carbohydrate slightly increased with the average concentration of $128.1 \pm 29.8 \mathrm{mgC} / \mathrm{g} \mathrm{VSS}$; the decreased intracellular carbohydrate concentration was $89.7 \mathrm{mgC} / \mathrm{g}$ VSS during the whole reaction phase. These results showed that the intracellular carbohydrate was mainly used for biochemical metabolism during the 


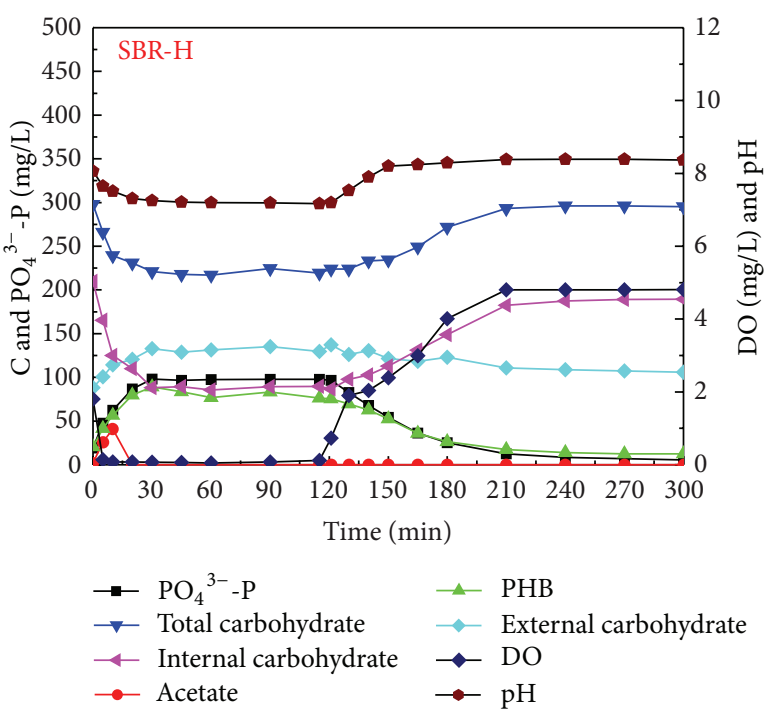

(a)

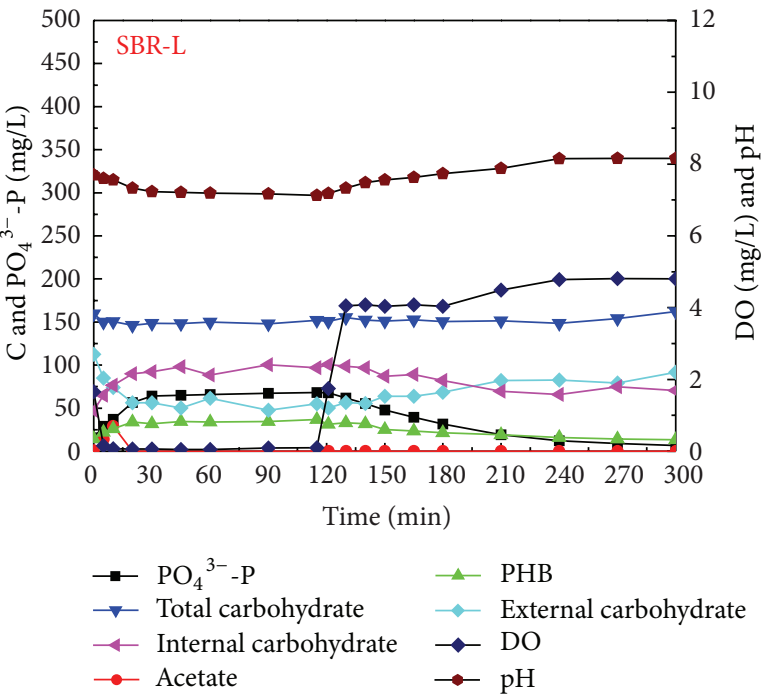

(b)

FIGURE 2: Dynamics of different parameters in typical cycles of SBR-H and SBR-L.

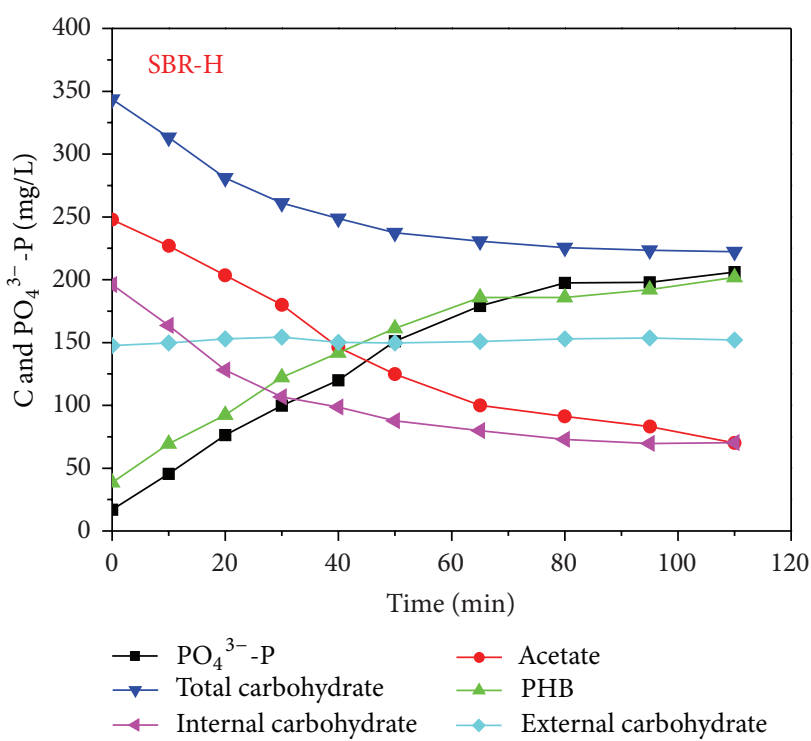

(a)

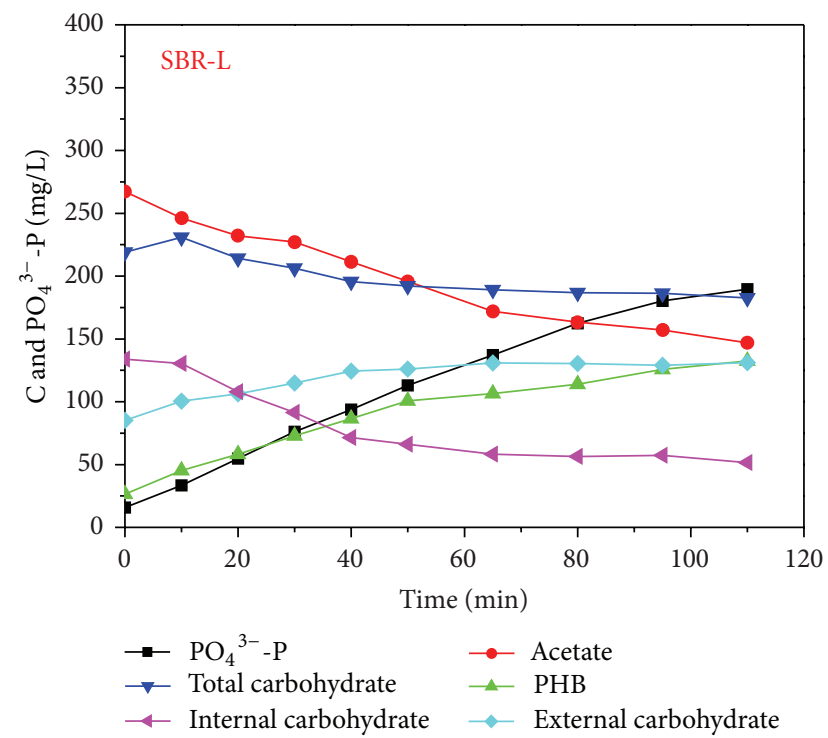

(b)

FIGURE 3: Anaerobic dynamics of polymers of PAOs with excess supply of organic carbons.

anaerobic phase, consistent with previous studies, such as that of $\mathrm{Wu}$ and Rodgers [20]. This also shows that it is necessary to differentiate the intracellular and extracellular carbohydrate for investigating dynamics of polymer in EBPR. For the extracellular carbohydrate, a slightly higher concentration of $128.3 \mathrm{mg} \mathrm{C} / \mathrm{g}$ VSS existed in SBR-L than that of $82.8 \mathrm{mg} \mathrm{C/g} \mathrm{VSS}$ in SBR-H. Extracellular carbohydrate is one main component of EPS $[17,18]$, and the above results showed that a low influent organic carbon condition induced a high extracellular carbohydrate production, which might be due to the fact that EPS production was a response to low nutrient condition as a protective mechanism for experiencing unfavourable low organic carbon conditions.
By comparing the regressed data in Table 1, during Phase A, $r_{\text {PHB-C/NaAc-C }}$ was similar in both reactors, indicating that the acetate could be taken up and stored as PHB efficiently, while, during Phase $\mathrm{B}$, a slightly high $r_{\mathrm{PHB}-\mathrm{C} / \mathrm{NaAc}-\mathrm{C}}$ value was obtained in SBR-H. In spite of a slight variation of $r_{\mathrm{Glycogen}-\mathrm{C} / \mathrm{NaAc}-\mathrm{C}}$, there was no significant difference in both reactors during both phases. During the whole reaction phase, $r_{\mathrm{PO} 4-\mathrm{P} / \mathrm{NaAc}-\mathrm{C}}$ in SBR-L was higher than that in SBR$\mathrm{H}$, indicating a high energy requirement from polyphosphate degradation in SBR-L, which showed that there might be high PAO activities or a higher number of PAOs in SBR-L than that in SBR-H. The calculated mole ratios between the released phosphorus and the utilized acetate were $0.40 \mathrm{~mol} \mathrm{P} / \mathrm{mol} \mathrm{C}$ in 
TABLE 1: Regressed biokinetic coefficients of polymers of PAOs under anaerobic conditions with the supply of excess organic carbon.

\begin{tabular}{lcccc}
\hline & \multicolumn{2}{c}{ Phase A: 0-50 min } & \multicolumn{2}{c}{ Phase B: 50-110 min } \\
& SBR-H & SBR-L & SBR-H & SBR-L \\
\hline$r_{\text {PHB-C/NaAc-C }}(\mathrm{mg} / \mathrm{mg})$ & $0.97(0.98)$ & $1.08(0.99)$ & $0.72(0.97)$ & $0.66(0.87)$ \\
$r_{\text {PO4-P/NaAc-C }}(\mathrm{mg} / \mathrm{mg})$ & $1.03(0.98)$ & $1.44(0.97)$ & $1.05(0.95)$ & $1.66(0.95)$ \\
$r_{\text {Glycogen-C/NaAc-C }}(\mathrm{mg} / \mathrm{mg})$ & $0.85(0.90)$ & $1.10(0.92)$ & $0.35(0.90)$ & $0.28(0.94)$ \\
\hline
\end{tabular}

TABLE 2: Regressed biokinetics of biomass or polymers by the first-order equation under both anaerobic and aerobic endogenous respirations $(1 / \mathrm{d})$.

\begin{tabular}{lcccc}
\hline & & SBR-H & & SBR-L \\
& Anaerobic & Aerobic & Anaerobic & $0.074(0.94)$ \\
SS & $0.074(0.98)$ & $0.070(0.98)$ & $0.038(0.82)$ & $0.082(0.95)$ \\
VSS & $0.041(0.86)$ & $0.048(0.89)$ & $0.029(0.56)$ & $0.058(0.90)$ \\
Total carbohydrate & $0.034(0.65)$ & $0.074(0.92)$ & $0.209(0.93)$ & $0.034(0.67)$ \\
Internal carbohydrate & $0.137(0.96)$ & $0.226(0.96)$ & $0.089(0.99)$ & $0.194(0.98)$ \\
Polyphosphate & $0.098(0.94)$ & $0.084(0.97)$ & & $0.091(0.99)$ \\
\hline
\end{tabular}

SBR-L and $0.28 \mathrm{~mol} \mathrm{P} / \mathrm{mol} \mathrm{C}$ in SBR-H during Phase A and were $0.14 \mathrm{~mol} \mathrm{P} / \mathrm{mol} \mathrm{C}$ and $0.16 \mathrm{~mol} \mathrm{P} / \mathrm{mol} \mathrm{C}$ during Phase B. During Phase A, a high reaction rate existed due to the existence of high external organic carbons, and the calculated polyphosphate requirement was in the theoretical range of 0.25-0.75 mol P/mol C [12], while during Phase $\mathrm{B}$, due to the limitation of intracellular carbohydrate and the slow activity of PAOs, the biokinetics were relatively slow and were different from those of Phase A.

3.3. Dynamics of Polymers of PAOs during Anaerobic and Aerobic Endogenous Respirations. Under anaerobic and aerobic endogenous respiration conditions, dynamics of polymers of PAOs and sludge concentrations are shown in Figure 4.

Under starve conditions, microorganisms will experience endogenous respiration $[8,21]$, and except the reduction in the biomass concentration, consumption of polymers (for PAOs, glycogen, $\mathrm{PHB}$, etc.) also occurs to provide the required maintenance energy. In SBR-H, during the anaerobic endogenous respiration, different utilization modes of polyphosphate and glycogen occurred, with the main utilization of glycogen initially and after 48 hours, with a quick utilization of polyphosphate. During the 168 hours of anaerobic endogenous respiration, glycogen decreased from $99.1 \mathrm{mg} \mathrm{C} / \mathrm{g}$ VSS to $34.3 \mathrm{mg} \mathrm{C} / \mathrm{g}$ VSS in SBR-H and the phosphorus released was $60.2 \mathrm{mg} \mathrm{P/g} \mathrm{VSS}$, while in SBR-L, glycogen decreased from $115.5 \mathrm{mg} \mathrm{C} / \mathrm{g}$ VSS to $21.2 \mathrm{mg} \mathrm{C/g} \mathrm{VSS}$ and the phosphorus released was $91.8 \mathrm{mg} \mathrm{P} / \mathrm{g}$ VSS. During the 168 hours of aerobic endogenous respiration, glycogen decreased from $99.6 \mathrm{mg}$ C/g VSS to $23.7 \mathrm{mg} \mathrm{C} / \mathrm{g}$ VSS in SBR$\mathrm{H}$ and the phosphorus released was $52.7 \mathrm{mg} \mathrm{P} / \mathrm{g} \mathrm{VSS}$, while in SBR-L, glycogen decreased from $115.0 \mathrm{mg} \mathrm{C} / \mathrm{g}$ VSS to $38.7 \mathrm{mg} \mathrm{C} / \mathrm{g}$ VSS in SBR-H and the phosphorus released was $89.3 \mathrm{mg}$ P/g VSS. These results showed that, during endogenous respiration, PAOs acclimated under the low influent organic carbon conditions utilized a higher amount of polymers than those of PAOs acclimated under a high influent acetate concentration.
During the anaerobic endogenous respiration, when the glycogen concentration decreased to $67.1 \mathrm{mg} \mathrm{C/g}$ VSS in SBR$\mathrm{H}$ and $85.4 \mathrm{mg} \mathrm{C/g} \mathrm{VSS} \mathrm{in} \mathrm{SBR-L,} \mathrm{degradation} \mathrm{of} \mathrm{polyphos-}$ phate occurred rapidly while this process was inhibited therebefore. During the aerobic endogenous respiration, when the glycogen concentration decreased to $81.8 \mathrm{mg} \mathrm{C/g} \mathrm{VSS} \mathrm{in} \mathrm{SBR-}$ $\mathrm{H}$ and $87.2 \mathrm{mg} \mathrm{C} / \mathrm{g}$ VSS in SBR-L, degradation of polyphosphate also occurred significantly with a high increase in the liquid phosphate concentration. These results showed that degradation of polyphosphate during endogenous respiration was controlled by the glycogen concentration and glycogen was firstly utilized as the energy source. When the glycogen concentration reached around $80 \pm 10 \mathrm{mg} \mathrm{C} / \mathrm{g}$ VSS, degradation of polyphosphate occurred. These showed that PAOs might prefer to utilize glycogen during the endogenous respiration for maintenance purposes $[8,20,22,23]$ and then utilize polyphosphate when the glycogen decreased to a certain level. Lopez et al. [8] also found that during the aerobic endogenous respiration, PAOs utilized PHA, glycogen, and polyphosphate sequentially, and phosphate release occurred after 16 hours of aerobic endogenous respiration.

For dynamics of all parameters during endogenous respiration, they were regressed by the first-order degradation equation and the results are shown in Table 2. There was no significant difference for biomass decay under anaerobic and aerobic conditions, which were close to those of $0.015-$ $0.0321 / \mathrm{d}$ obtained by $\mathrm{Lu}$ et al. [23], while they were lower than those of $0.14-0.151 / \mathrm{d}$ obtained in other studies $[8,20]$. It should be noticed that, in most studies, biomass during decay was represented by the decrease in the concentration of VSS, while different environmental conditions might induce different intracellular or extracellular polymer concentrations, which might affect the description of biomass decay by using the parameter of VSS. Compared with the decay of biomass, decay coefficients of intracellular polymers were much higher. In addition, the degradation coefficient of glycogen was higher than that of polyphosphate. These results showed that during endogenous respiration, microorganisms 


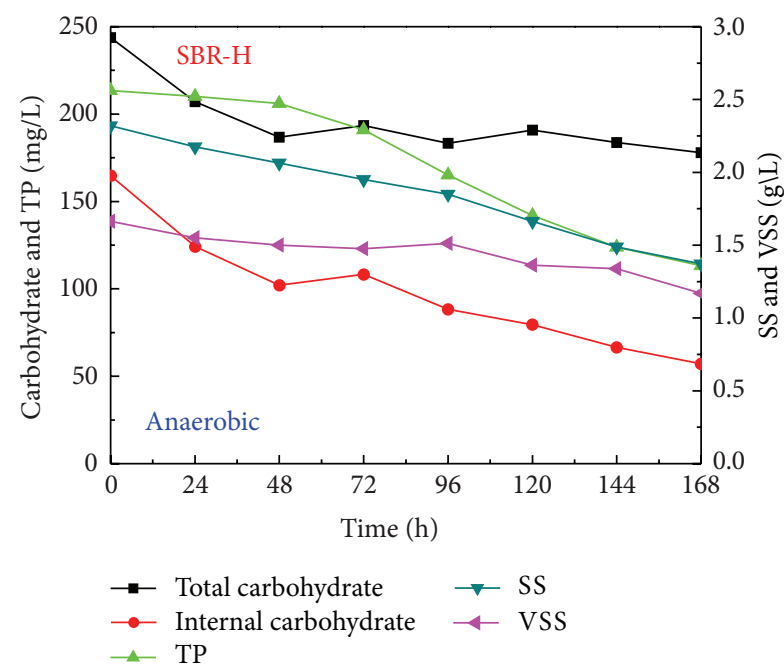

(a)

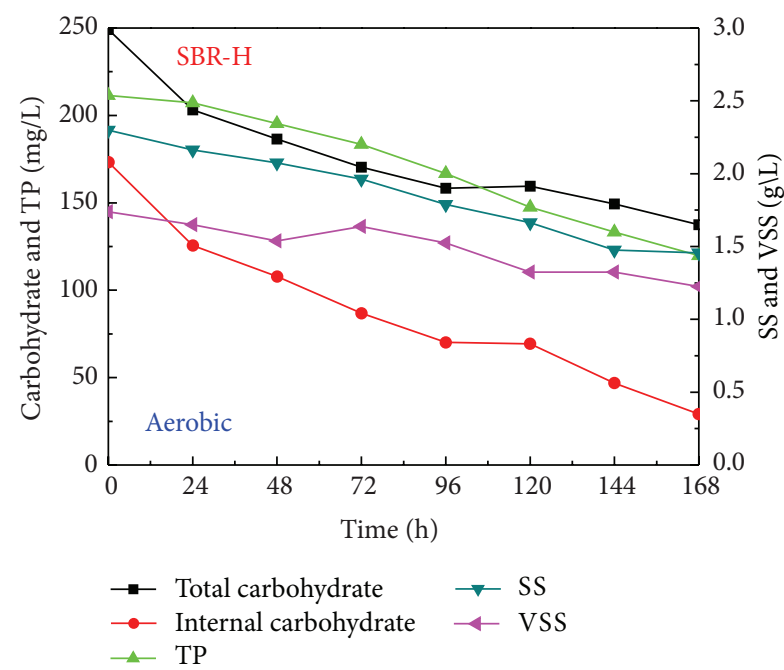

(c)

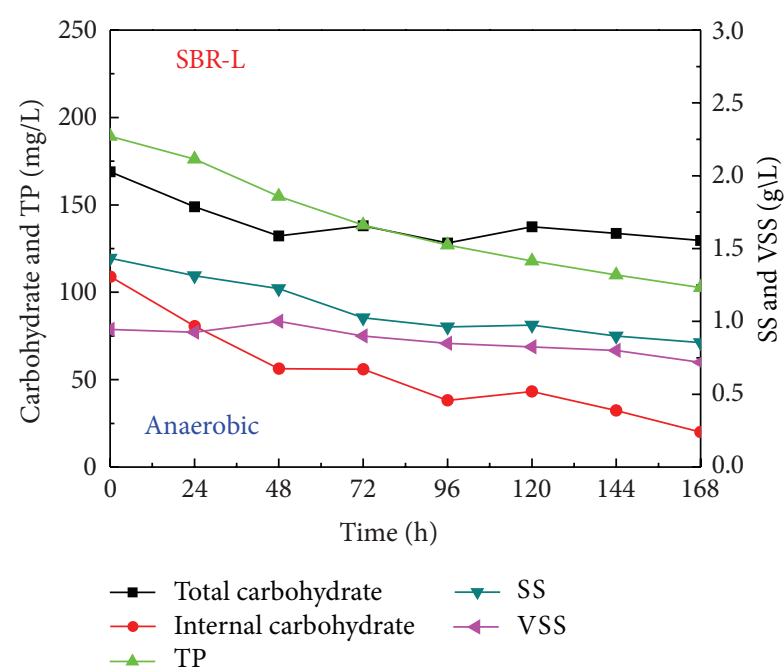

(b)

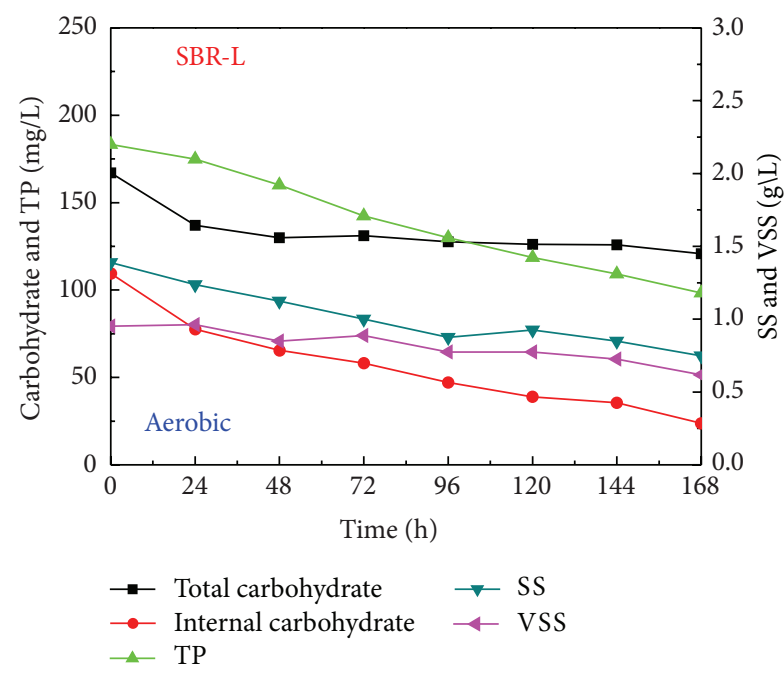

(d)

FIGURE 4: Dynamics of polymers of PAOs and sludge concentrations during anaerobic and aerobic endogenous respirations.

would prefer to utilize stored polymers to maintain their activities. Comparing between degradation coefficients during both anaerobic and aerobic endogenous respirations, it was surprising to see there was not much difference for both decay of biomass and polymers.

During the anaerobic endogenous respiration, the degradation of intracellular carbohydrate in SBR-L was higher than that in SBR-H, which could be due to a slightly earlier utilization of polyphosphate in SBR-H and this might retard the utilization of glycogen, while under the aerobic endogenous respiration, the degradation of intracellular carbohydrate was slightly lower in SBR-L than that in SBR-H, which could be due to the fact that PAOs in SBR-L experienced starve conditions longer than those in SBR-H during acclimation, and they might had been used to the starve conditions better. For carbohydrate, there was a significant difference in biokinetics by using the total carbohydrate or the intracellular carbohydrate, confirming results from the previous study of Wu and Rodgers [20]. The anaerobic intracellular carbohydrate degradation rate was lower than that obtained by $\mathrm{Wu}$ and Rodgers [20], while similar results were obtained during the aerobic endogenous respiration. In the present study, PAOs were acclimated and all experiments were carried out at $25^{\circ} \mathrm{C}$, while PAOs are psychrophile $[24,25]$. The high temperature used in the present study might cause the decreased biokinetic activities, including a low anaerobic intracellular degradation coefficient.

\section{Conclusions}

Dynamics of polymers of PAOs under different organic carbon concentrations, including typical cycles, anaerobic metabolism with supply of excess organic carbon, and both anaerobic and aerobic endogenous respiration, were examined. Influent organic carbon concentrations had little influence on the yield of PAOs and the anaerobic polyphosphate release, 
while a low influent organic carbon concentration favoured the acclimation of PAOs and induced a high production of extracellular carbohydrate. During both anaerobic and aerobic endogenous respirations, when glycogen decreased to below around $80 \pm 10 \mathrm{mg} \mathrm{C/g} \mathrm{VSS}$, PAOs began to utilize polyphosphate significantly. Regressed by the first-order reaction model, the degradation of glycogen possessed the highest reaction rate and was then followed by polyphosphate, while biomass decay had the lowest reaction rate.

\section{Conflict of Interests}

The authors declare that there is no conflict of interests regarding the publication of this paper.

\section{Acknowledgments}

This study was supported by the National Natural Science Foundation of China (51108242) and the Scientific Research Foundation for the Returned Overseas Chinese Scholars, State Education Ministry, China.

\section{References}

[1] T. Mino, M. C. M. Van Loosdrecht, and J. J. Heijnen, "Microbiology and biochemistry of the enhanced biological phosphate removal process," Water Research, vol. 32, no. 11, pp. 3193-3207, 1998.

[2] H. G. Schlegel, General Microbiology, Cambridge University Press, Cambridge, UK, 1993.

[3] L. S. Serafim, P. C. Lemos, C. Levantesi, V. Tandoi, H. Santos, and M. A. M. Reis, "Methods for detection and visualization of intracellular polymers stored by polyphosphate-accumulating microorganisms," Journal of Microbiological Methods, vol. 51, no. 1, pp. 1-18, 2002.

[4] A. R. Pitman, S. L. V. Venter, and H. A. Nicholls, "Practical experience with biological phosphorus removal plants in Johannesburg," Water Science and Technology, vol. 15, no. 3-4, pp. 233259, 1983.

[5] H. Miyake and E. Morgenroth, "Optimization of enhanced biological phosphorus removal after periods of low loading," Water Environment Research, vol. 77, no. 2, pp. 117-127, 2005.

[6] C. H. Ahn, H.-D. Park, and J. K. Park, "Enhanced biological phosphorus removal performance and microbial population changes at high organic loading rates," Journal of Environmental Engineering, vol. 133, no. 10, pp. 962-969, 2007.

[7] C. H. Ahn, J. K. Park, and K. S. Kim, "Microbial adaptability to organic loading changes in an enhanced biological phosphorus removal process," Journal of Environmental Engineering, vol. 132, no. 8, pp. 909-917, 2006.

[8] C. Lopez, M. N. Pons, and E. Morgenroth, "Endogenous processes during long-term starvation in activated sludge performing enhanced biological phosphorus removal," Water Research, vol. 40, no. 8, pp. 1519-1530, 2006.

[9] G. Yilmaz, R. Lemaire, J. Keller, and Z. Yuan, "Effectiveness of an alternating aerobic, anoxic/anaerobic strategy for maintaining biomass activity of BNR sludge during long-term starvation," Water Research, vol. 41, no. 12, pp. 2590-2598, 2007.

[10] R. Brun, M. Kühni, H. Siegrist, W. Gujer, and P. Reichert, "Practical identifiability of ASM2d parameters-systematic selection and tuning of parameter subsets," Water Research, vol. 36, no. 16, pp. 4113-4127, 2002.

[11] L. Tijhuis, M. C. M. Van Loosdrecht, and J. J. Heijnen, "A thermodynamically based correlation for maintenance Gibbs energy requirements in aerobic and anaerobic chemotrophic growth," Biotechnology and Bioengineering, vol. 42, no. 4, pp. 509-519, 1993.

[12] G. J. F. Smolders, J. Van der Meij, M. C. M. Van Loosdrecht, and J. J. Heijnen, "Model of the anaerobic metabolism of the biological phosphorus removal process: stoichiometry and $\mathrm{pH}$ influence," Biotechnology and Bioengineering, vol. 43, no. 6, pp. 461-470, 1994.

[13] APHA, AWWA, and WPCF, Standard Methods for the Examination of Water and Wastewater, American Public Health Association, Washington, DC, USA, 19th edition, 1995.

[14] D. B. Karr, J. K. Waters, and D. W. Emerich, "Analysis of poly- $\beta$-hydroxybutyrate in Rhizobium japonicum bacteroids by ion-exclusion high-pressure liquid chromatography and UV detection," Applied and Environmental Microbiology, vol. 46, no. 6, pp. 1339-1344, 1983.

[15] M. Rodgers and G. Wu, "Production of polyhydroxybutyrate by activated sludge performing enhanced biological phosphorus removal," Bioresource Technology, vol. 101, no. 3, pp. 1049-1053, 2010.

[16] A. Lanham, A. Ricardo, M. Coma et al., "Optimisation of glycogen quantification in mixed microbial cultures," Bioresource Technology, vol. 118, pp. 518-525, 2012.

[17] X. Y. Li and S. F. Yang, "Influence of loosely bound extracellular polymeric substances (EPS) on the flocculation, sedimentation and dewaterability of activated sludge," Water Research, vol. 41, no. 5, pp. 1022-1030, 2007.

[18] D. T. Sponza, "Extracellular polymer substances and physicochemical properties of flocs in steady- and unsteady-state activated sludge systems," Process Biochemistry, vol. 37, no. 9, pp. 983-998, 2002.

[19] Y. Tu and A. J. Schuler, "Low acetate concentrations favor polyphosphate-accumulating organisms over glycogen-accumulating organisms in enhanced biological phosphorus removal from wastewater," Environmental Science and Technology, vol. 47, pp. 3816-3824, 2013.

[20] G. Wu and M. Rodgers, "Dynamics and function of intracellular carbohydrate in activated sludge performing enhanced biological phosphorus removal," Biochemical Engineering Journal, vol. 49, no. 2, pp. 271-276, 2010.

[21] X.-D. Hao, Q.-L. Wang, J.-Y. Zhu, and M. C. M. Van Loosdrecht, "Microbiological endogenous processes in biological wastewater treatment systems," Critical Reviews in Environmental Science and Technology, vol. 40, no. 3, pp. 239-265, 2010.

[22] H. Satoh, T. Mino, and T. Matsuo, "Uptake of organic substrates and accumulation of polyhydroxyalkanoates linked with glycolysis of intracellular carbohydrates under anaerobic conditions in the biological excess phosphate removal processes," Water Science and Technology, vol. 26, no. 5-6, pp. 933-942, 1992.

[23] H. Lu, J. Keller, and Z. Yuan, "Endogenous metabolism of Candidatus Accumulibacter phosphatis under various starvation conditions," Water Research, vol. 41, no. 20, pp. 4646-4656, 2007.

[24] U. G. Erdal, Z. K. Erdal, and C. W. Randall, "The competition between PAOs (phosphorus accumulating organisms) and GAOs (glycogen accumulating organisms) in EBPR (enhanced 
biological phosphorus removal) systems at different temperatures and the effects on system performance," Water Science and Technology, vol. 47, no. 11, pp. 1-8, 2003.

[25] C. M. López-Vázquez, C. M. Hooijmans, D. Brdjanovic, H. J. Gijzen, and M. C. M. van Loosdrecht, "Factors affecting the microbial populations at full-scale enhanced biological phosphorus removal (EBPR) wastewater treatment plants in The Netherlands," Water Research, vol. 42, no. 10-11, pp. 2349-2360, 2008. 

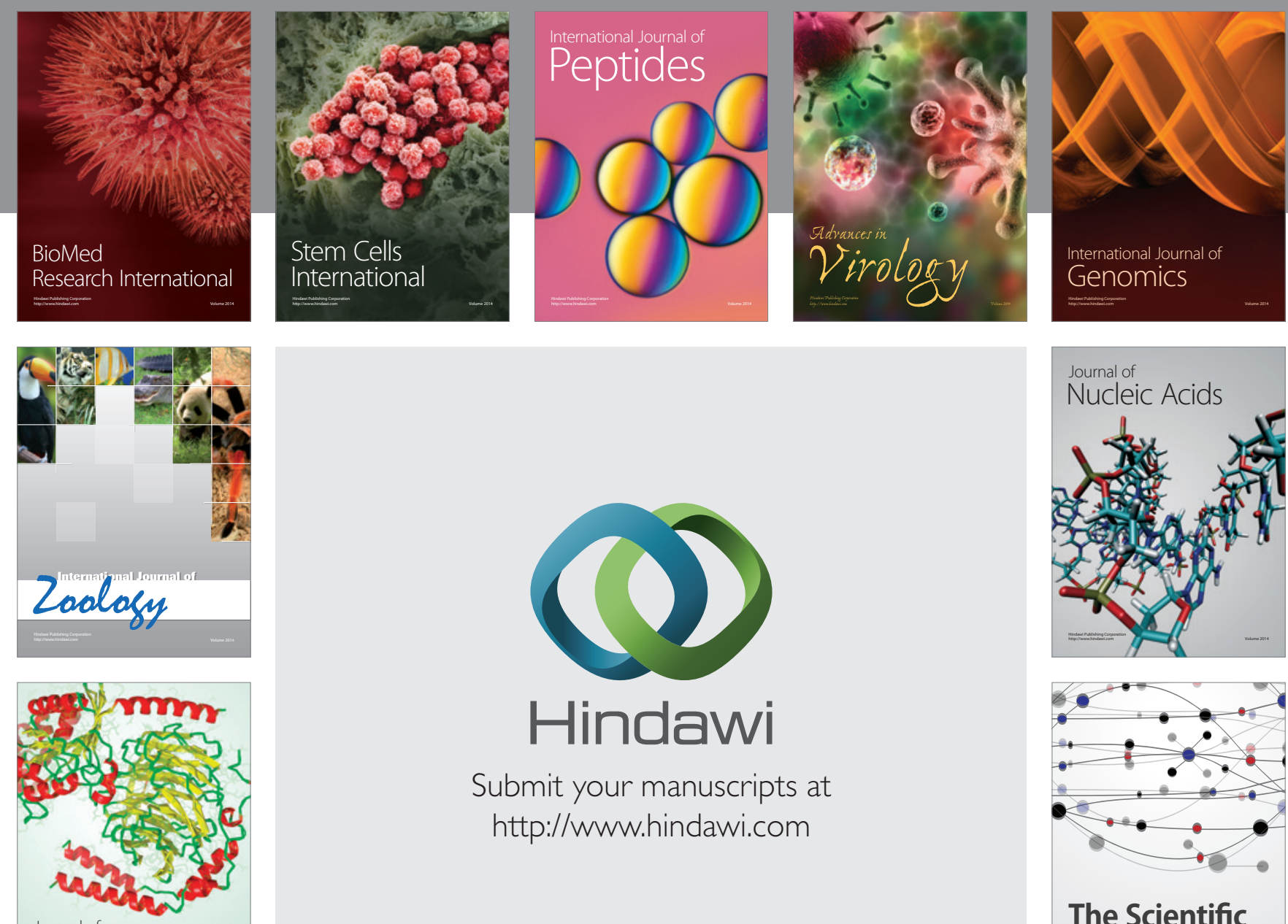

Submit your manuscripts at

http://www.hindawi.com

Journal of
Signal Transduction
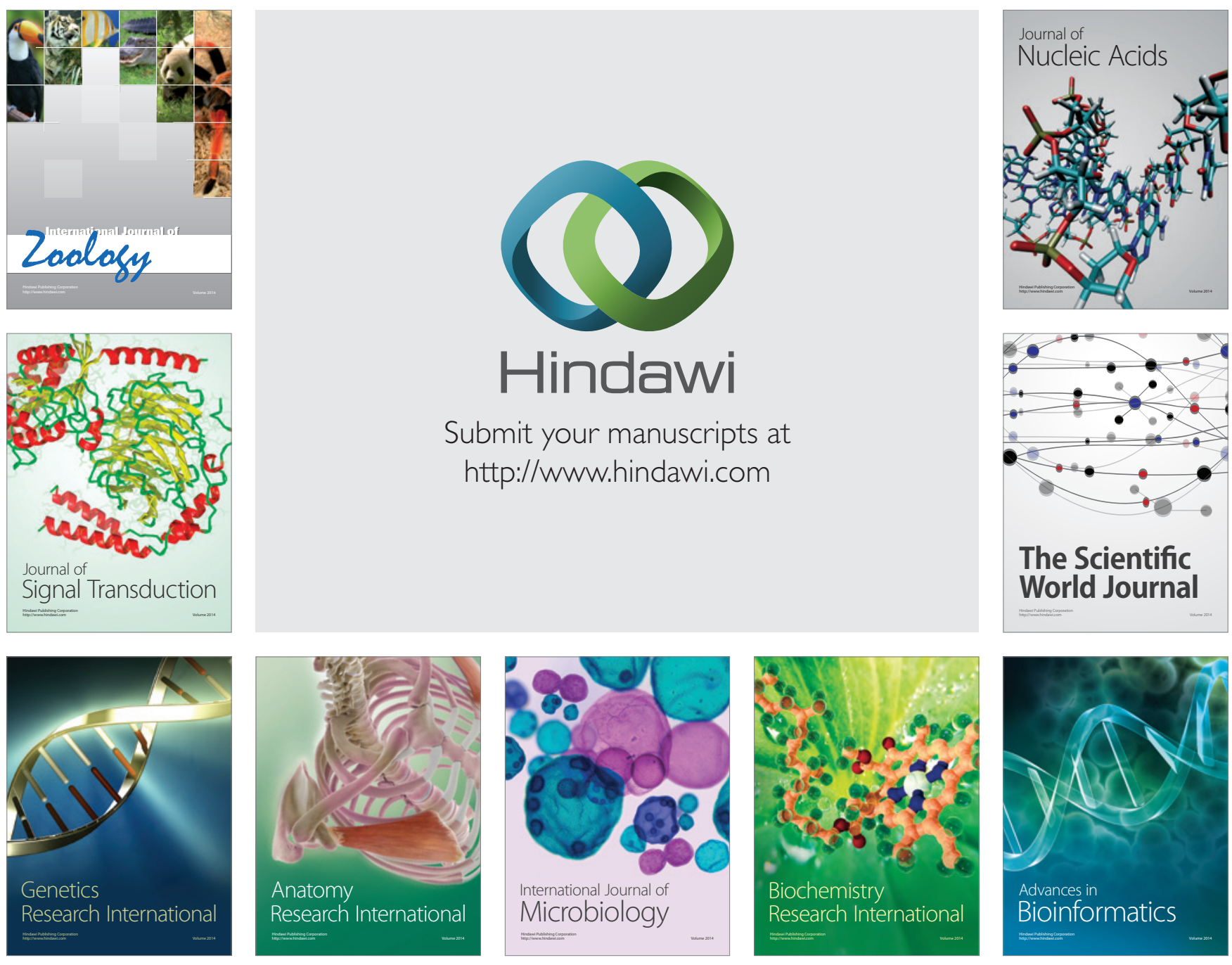

The Scientific World Journal
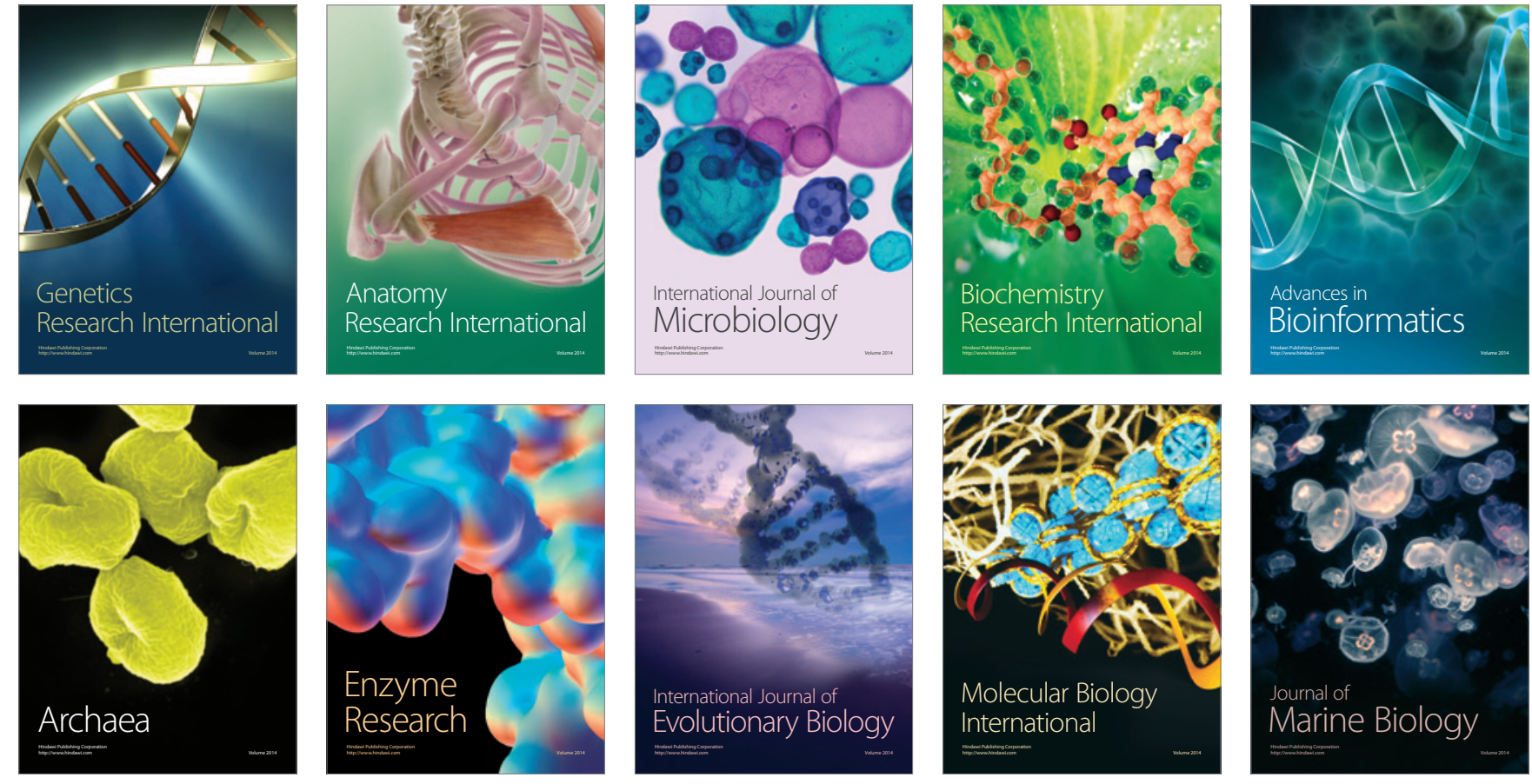